\title{
Oportunidades de la epigenética como enfoque para el tratamiento del cáncer de mama
}

\author{
Epigenetics opportunities as an approach to breast cancer \\ treatment. Epi-pharmaceuticals for breast cancer \\ Martha Edith Macías Pérez, ${ }^{*}$ Rolando Alberto Rodríguez-Fonseca, ${ }^{\ddagger}$ \\ Elvia Mera Jiménez, ${ }^{*}$ Maricarmen Hernández Rodríguez*
}

* Laboratorio de cultivo celular de la sección de estudios de postgrado e investigación. Escuela Superior de Medicina, Instituto Politécnico Nacional.

‡ Unidad de Investigación Biomédica de Zacatecas (UIBMZ) del Instituto Mexicano del Seguro Social (IMSS).

Correspondencia:

Dra. Martha Edith

Macías Pérez

Laboratorio de cultivo

celular de la sección de

estudios de Postgrado e

Investigación.

Escuela Superior de Medi-

cina, Instituto Politécnico

Nacional,

Plan de San Luis y Díaz

Mirón s/n,

Col. Casco de Santo Tomas, 11340,

Alcaldía Miguel Hidalgo,

Ciudad de México.

Teléfono: (55) 5729-6000,

ext. 62767

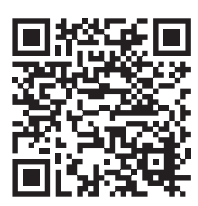

\section{RESUMEN}

El cáncer de mama representa 16\% de todos los cánceres femeninos en el mundo y la mayoría (69\%) de las defunciones por esta causa se registran en los países en desarrollo. De acuerdo con lo reportado por la OMS, a nivel mundial, surgen 1.38 millones de nuevos casos y fallecen 458 mil personas cada año. El cáncer de mama es la segunda causa de muerte en mujeres mexicanas mayores de 20 años de edad. El conocimiento de las ciencias ómicas y de nuevos niveles de organización en la expresión genética, como es el caso de la epigenética, permiten plantear nuevas estrategias terapéuticas para los pacientes con cáncer de mama. En este sentido, el desarrollo de un tratamiento personalizado que permita modular las vías de señalización relacionadas con la supervivencia del tumor resulta prometedor y paralelamente plantea preocupación por los posibles efectos adversos. El propósito de esta revisión es identificar las lecciones aprendidas en el tratamiento epigenético del cáncer de mama, así como los principales tratamientos epigenéticos de uso clínico, los aspectos adversos identificados y las áreas de oportunidad para mejorar el abordaje terapéutico con fármacos epigenéticos.

Palabras clave: Cáncer de mama, epigenética, tratamiento personalizado, fármacos epigenéticos.

\section{ABSTRACT}

Breast cancer represents $16 \%$ of all female cancers in the world, and the majorities (69\%) of deaths from this cause are registered in developing countries. According to WHO, each year in the world, 1.38 million new cases appear, and 458 thousand people die. It is the second leading cause of death in Mexican women over 20 years old. The knowledge of omic sciences and new levels of organization in gene expression, such as epigenetics, allow to propose new treatment strategies in breast cancer patients, to generate a personalized treatment specific to the tumor, modulating with a single treatment multiple signaling pathways involved in key processes for tumor survival, although this may be promising, it also raises different views on the adverse effects and drug interactions of epigenetic drugs. The purpose of this review is to identify the lessons learned in the breast cancer epigenetic treatment. The main epigenetic treatments for clinical use, the adverse aspects identified and the opportunity to improve the therapeutic approach with epigenetic drugs.

Keywords: Breast cancer, epigenetics, personalized treatment, epigenetics drugs.

Citar como: Macías PME, Rodríguez-Fonseca RA, Mera JE, Hernández RM. Oportunidades de la epigenética como enfoque para el tratamiento del cáncer de mama. Rev Mex Mastol. 2020; 10 (3): 93-97. https://dx.doi.org/10.35366/99158 


\section{INTRODUCCIÓN}

Clasificación general del cáncer de mama

El cáncer de mama se ha clasificado a nivel histológico, molecular y por etapas o estadios. Histológicamente existen dos tipos principales: carcinoma invasivo ductal y carcinoma invasivo lobular. ${ }^{1}$ A nivel molecular se han reportado tres subtipos de tumor de acuerdo a la expresión de receptor de estrógeno $(\mathrm{ERa})$, receptor de progesterona (PR) y a la amplificación del gen HER2 (human epidermal growth factor 2), también llamado ERBB2 o HER2/neu, con los cuales se obtienen las siguientes combinaciones de tumores mamarios: hormono receptor positivo/ HER2-, hormono receptor negativo/HER2+ y triple negativo para aquellos tumores que no expresan ninguno de los tres marcadores moleculares. ${ }^{2-5}$ La incidencia de cada uno de estos tumores depende de múltiples factores; pero usualmente la mayor incidencia se encuentra en los tumores ERa $<$ PR $<$ HER2 + . De igual forma, el pronóstico puede variar de acuerdo a varios factores; no obstante, de mayor a menor pronóstico, en general, podemos encontrar $\mathrm{ERa}<\mathrm{PR}<\mathrm{HER} 2+$, siendo los tumores HER2 + los del pronóstico más bajo, mientras que los tumores triple negativo al desconocerse su fisiopatología molecular hacen más complicado el proceso de elegir los tratamientos más adecuados. ${ }^{3}$
Selección de la estrategia de tratamiento de acuerdo al subtipo de cáncer de mama

Para el cáncer de mama no metastásico existen dos tipos de tratamiento: sistémico y local. Éste último consiste en la resección quirúrgica del tumor y la resección o reubicación de los ganglios linfáticos axilares, posterior a la resección quirúrgica se prescribe radioterapia. Por otra parte, el tratamiento sistémico se subdivide en tratamiento adyuvante (postoperatorio) y en tratamiento neoadyuvante (preoperatorio); el tratamiento sistémico también puede prescribirse antes y después de la resección quirúrgica. $^{3}$

El esquema terapéutico y la elección de fármacos se hacen con base en los tres marcadores moleculares previamente mencionados y del estadio del cáncer mamario que presenta el paciente. No obstante, los tratamientos pueden modificarse dependiendo de las características específicas del paciente como el estadio anatómico del cáncer en el paciente, la presencia o ausencia de metástasis, comorbilidades, recidiva, curso clínico, entre otros. ${ }^{5}$

\section{DESARROLLO DE NUEVOS TRATAMIENTOS PARA EL CÁNCER DE MAMA CON BASE EN LAEPIGENÉTICA DEL TUMOR}

Los cambios epigenéticos como la metilación del ADN, las modificaciones de las histonas

Tabla 1: Epifármacos aprobados por la FDA para estudios clínicos contra cáncer de mama.

\begin{tabular}{|c|c|c|c|}
\hline Nombre & Estudio clínico/año & Indicación & Blanco farmacológico \\
\hline $\begin{array}{l}\text { Azacitidina } \\
\text { Entinostat }\end{array}$ & NCT01349959/2020 & $\begin{array}{l}\text { Cáncer de mama avanzado (IIIC y IV) } \\
\text { triple negativo y hormono refractario }\end{array}$ & $\begin{array}{l}\text { Inhibidor del ADN metiltransfera- } \\
\text { sa (DNMTi), HDAC1 y HDAC3 }{ }^{13-15}\end{array}$ \\
\hline $\begin{array}{l}\text { Ácido valproico } \\
\text { FEC } 100\end{array}$ & NCT01010854/2018 & $\begin{array}{l}\text { Cáncer de mama metastásico prima- } \\
\text { rio o localmente avanzado }\end{array}$ & HDAC1 y $2^{15,16}$ \\
\hline $\begin{array}{l}\text { MK0683 } \\
\text { Vorinostat } \\
\text { Suberoylanilide } \\
\text { hydroxamic acid (SAHA) }\end{array}$ & NCT00126451/2017 & $\begin{array}{l}\text { Cáncer de mama, colorrectal y de } \\
\text { pulmón de células no pequeñas } \\
\text { recidivante o refractario }\end{array}$ & HDAC clase 1 y $2^{15,17}$ \\
\hline $\begin{array}{l}\text { Tamoxifeno } \\
\text { Vorinostat } \\
\text { Pembrolizumab }\end{array}$ & NCT02395627/2020 & $\begin{array}{l}\text { Cáncer de mama resistente a la } \\
\text { terapia hormonal }\end{array}$ & HDAC1 y $3^{15,18}$ \\
\hline $\begin{array}{l}\text { Entinostat } \\
\text { Atezolizumab }\end{array}$ & NCT02708680/2018 & Cáncer de mama & HDAC1 y $3^{15}$ \\
\hline
\end{tabular}


y la expresión anormal de los ARN no codificantes se han considerado nuevos biomarcadores en el diagnóstico, terapia y prevención del cáncer de mama. ${ }^{6}$ Sin embargo, para lograr mejores resultados es preciso conocer las particularidades del tumor de cada paciente y con ello usar como estrategia de tratamiento un enfoque personalizado; para esto, además de conocer las características fisiopatológicas del tumor, también se requiere conocer información compleja como el fenotipo y el paisaje de expresión genética y epigenética del tejido tumoral. El entendimiento de la complejidad del tumor $y$, en especial, de su epigenética, es el fundamento para el desarrollo de nuevos tratamientos para el cáncer de mama. ${ }^{7-9}$

Fármacos epigenéticos de uso clínico para cáncer de mama

Los primeros fármacos epigenéticos (epifármacos) aprobados por la Food and Drug Administration (FDA) para el tratamiento contra el cáncer son los fármacos para revertir la metilación del ADN. La azacitidina es un inhibidor del ADN metiltransferasa, utilizado para el tratamiento del síndrome preleucémico mielodisplásico (MDS). ${ }^{10}$ Por otra parte, los fármacos epigenéticos que actúan como inhibidores de las histonas desacetilasas (HDACi) también han sido aprobados por la FDA para el tratamiento de diferentes tipos de cáncer (mieloma múltiple, linfoma cutáneo de células T, etcétera) $i^{11}$ no obstante, este tipo de fármacos se administran en combinación con otros agentes terapéuticos a fin de potenciar el efecto terapéutico y de reducir los efectos adversos. ${ }^{12}$ Tanto los inhibidores de la metilación del ADN como los HDACi han sido aprobados por la FDA para el tratamiento de tumores sólidos. La Tabla 1 enlista algunos de los estudios clínicos aprobados por la FDA en los que se utilizan algunos epifármacos en combinación con la quimioterapia para conseguir potenciar los efectos benéficos en los pacientes, reducir los tumores o aumentar la respuesta inmune de los pacientes ante el cáncer de mama, así como determinar dosis de administración y evaluar los efectos adversos.
La mayoría de los estudios reportados en esta revisión se encuentran en fase II de investigación clínica; así como estos, existe una gran cantidad de epifármacos que continúan en desarrollo y en diferentes fases de investigación clínica y preclínica. Cabe resaltar que, dentro de la información proporcionada por estos estudios, se ha mencionado que los efectos secundarios observados no son mayores, ni más severos o graves que los reportados por los agentes quimioterapéuticos con los que se administraron, lo cual abre la posibilidad de encontrar epifármacos adecuados al tipo de cáncer de cada paciente para favorecer el efecto de los agentes quimioterapéuticos sin agravar el estado del paciente.

Azacitidina y entinostat como tratamiento combinado para cáncer de mama avanzado, estudio clínico fase 2

El tratamiento con azacitidina en líneas celulares de cáncer de mama, colorrectal y ovárico ha mostrado modulación positiva de la señalización del interferón, el procesamiento y la presentación de antígenos y las citocinas/ quimiocinas. ${ }^{13}$ Las muestras de biopsias de pacientes seleccionadas mostraron una regulación positiva de los genes marcadores informativos de ascendencia (AIM; polimorfismos en un nucleótido que expresa diferencias entre distintas poblaciones que permiten estimar la ascendencia de un individuo) después del tratamiento con terapia epigenética. Estos resultados apuntan a un amplio papel inmunoestimulador de los fármacos desmetilantes del ADN en cáncer de mama, colorrectal y ovárico. ${ }^{13}$

Entinostat, a base de benzamida, inhibe las HDAC-1 y HDAC-3, dando como resultado la inhibición del crecimiento de las células tumorales de varios tipos de cáncer, por lo que la administración de azacitidina junto con entinostat puede destruir más células tumorales. ${ }^{13,14}$ En el estudio fase II (NCT01349959) se incluyeron pacientes con cáncer de mama triple negativo (TNBC) y cáncer de mama hormono resistente (HRBC). La inmunomodulación positiva observada sugiere la posibilidad de que la terapia epigenética pueda sensibilizar a los pacientes con cáncer a la inmunoterapia (Tabla 1). ${ }^{15}$ 
FEC 100 y ácido valproico como tratamiento para cáncer primario metastásico, estudio clínico fase 2

En el estudio fase I (NCT01010854) se observó que el ácido valproico en combinación con FEC 100 (combinación de 5-fluorouracilo, epirubicina y ciclofosfamida para el tratamiento de cáncer de mama) no produce más efectos adversos que los comúnmente producidos por la quimioterapia, por lo que se consideró que podría ser una combinación que podría aumentar la eficacia de la quimioterapia. Esta combinación se sometió a un estudio fase II en pacientes con tumor mamario, ganglios linfáticos afectados o pacientes cuyo tumor se ha diseminado. Aunque no mostró efectos adversos, tampoco mostró mejora en la eficacia del tratamiento, por lo que se suspendió el avance de los estudios (Tabla 1). ${ }^{15,16}$

MK0683 vorinostat (suberoylanilide hydroxamic acid [SAHA]) en cáncer de mama, colorrectal y de pulmón de células no pequeñas recidivante o refractario, estudio clínico

Vorinostat (Zolinza) es un inhibidor de HDAC-1 y HDAC-2 fue sometido a un ensayo multicéntrico de fase II (NCT00126451) en pacientes con cáncer de mama o de pulmón no microcítico medible para evaluar la tasa de respuesta, la seguridad y la tolerabilidad. A dosis tóxicas de 400 o 300 mg se observó anorexia, astenia, náuseas, trombocitopenia, vómitos y pérdida de peso. No se observó DLT (Doses-Limiting Toxicity). DLT se define como un evento adverso clínicamente significativo o un valor de laboratorio anormal, evaluado como no relacionado con la progresión de la enfermedad intercurrente o medicamentos concomitantes de acuerdo con el National Cancer Institute-Common Terminology Criteria for Adverse Events (NCICTCAE, por sus siglas en inglés) al nivel de 200 mg dos veces al día. Se observó estabilización de la enfermedad en ocho pacientes, pero no hubo respuestas confirmadas. Once pacientes interrumpieron el tratamiento debido a efectos adversos. El vorinostat en un programa oral diario durante 14 días/tres semanas fue tolerable a $200 \mathrm{mg}$ dos veces al día solamente, y no se observaron respuestas en este estudio. Sin embargo, la mayoría de los pacientes tuvieron una exposición limitada al fármaco que no permitió un análisis de eficacia confiable (Tabla 1). ${ }^{15-17}$

Tamoxifeno, vorinostat y pembrolizumab en cáncer de mama resistente a la terapia hormonal, estudio clínico fase 2

Este es el primer estudio clínico (NCT02395627) que analiza la combinación de vorinostat un inhibidor de histonas desacetilasas clases 1 y 2 , un antiestrogénico (tamoxifeno) y un inhibidor de proteína programada de muerte celular 1 (PD-1), pembrolizumab en pacientes pre- o postmenopáusicas con ERa + cáncer de mama avanzado con progresión en múltiples terapias previas. El objetivo de este estudio es demostrar que vorinostat puede aumentar la expresión de PD-1 y PD-L1. La respuesta objetiva fue de $4 \%$ y la tasa de beneficio clínico fue de 19\%. Se observó agotamiento (o envejecimiento precoz) de células T-CD4+ y depleción de células T reguladoras inducida por el tratamiento en $5 / 5$ pacientes con beneficio clínico, pero sólo en un no respondedor (PD-1+). La infiltración de linfocitos tumorales fue de $0.17 \%$. Sólo dos no respondedores tenían expresión de PD-L1> $1 \%$. Estos datos demuestran que los pacientes PD-L1 negativo con cáncer de mama ER positivo son quienes tienen más probabilidades de beneficiarse del punto de control inmune y la inhibición de HDAC (Tabla 1). ${ }^{15,18}$

Entinostat y atezolizumab en cáncer de mama avanzado triple negativo, estudio clínico fase 2

El propósito de este estudio es determinar la eficacia, seguridad y tolerabilidad del entinostat usado en combinación con atezolizumab en pacientes con cáncer de mama avanzado triple negativo (aTNBC). El estudio tiene dos fases: una fase de determinación de dosis de etiqueta abierta (fase 1b) seguida de una fase de expansión (fase 2). La fase de expansión evaluará la eficacia y seguridad del entinostat cuando se administra en el RP2D con atezolizumab en pacientes con aTNBC en un entorno 
aleatorizado, doble ciego, controlado con placebo. Los resultados de este estudio no fueron reportados (Tabla 1). ${ }^{15}$

\section{BIBLIOGRAFÍA}

1. Foulkes WD, Smith IE, Reis-Filho JS. Triple-negative breast cancer. N Engl J Med. 2010; 363: 1938-1948.

2. Corben AD. Pathology of invasive breast disease. Surg Clin North Am. 2013; 93 (2): 363-392.

3. Waks AG, Winer EP. Breast cancer treatment: a review. JAMA. 2019; 321 (3): 288-300.

4. Loibl S, Gianni L. HER2-positive breast cancer. Breast Cancer. 2017; 389 (10087): P2415-2429.

5. Wolff AC, Hammond MEH, Hicks DG, Dowsett M, McShane LM, Allison KH et al. Recommendations for Human Epidermal Growth Factor Receptor 2 Testing in Breast Cancer: American Society of Clinical Oncology/College of American Pathologists Clinical Practice Guideline Update. Journal of Clinical Oncology. 2013; 31: 3997-4013.

6. Ganesan A, Arimondo PB, Rots MG, Jeronimo C, Berdasco $M$. The timeline of epigenetic drug discovery: from reality to dreams. Clinical Epigenetics. 2019; 11: 174.

7. Ahuja N, Sharma AR, Baylin SB. Epigenetic therapeutics: a new weapon in the war against cancer. Annu Rev Med. 2016; 67: 73-89.

8. Lu Y, Chan YT, Tan HY, Li S, Wang N, Feng Y. Epigenetic regulation in human cancer: the potential role of epi-drug in cancer therapy. Molecular Cancer. 2020; 19: 79.

9. Denkert C, Liedtke C, Tutt A, Minckwitz Gv, Molecular alterations in triple-negative breast cancer the road to new treatment strategies. Breast Cancer. 2017; 389 (10087): P2430-2442.
10. Kaminskas E, Farrell AT, Wang YC. FDA drug approval summary: azacitidine 5-azacytidine, Vidaza for injectable suspension. Oncologist. 2005; 10: 176-182.

11. Bates SE. Epigenetic therapies for cancer. N Engl J Med. 2020; 383 (7): 650-663.

12. Mazzone R, Zwergel C, Mai A, Valente S. Epi-drugs in combination with immunotherapy: a new avenue to improve anticancer efficacy. Clinical Epigenetics. 2017; 9 (59).

13. Li H, Chiappinelli KB, Guzzetta AA, Easwaran H, Yen RWC, Vatapalli1 $\mathrm{R}$ et al. Immune regulation by low doses of the DNA methyltransferase inhibitor 5 -azacitidine in common human epithelial cancers. Oncotarget. 2014; 5: 587-598.

14. Bradner JE, West N, Grachan M, Greenberg EF, Haggarty SJ, Warnow $\mathrm{T}$ et al. Chemical phylogenetics of histone deacetylases. Nat Chem Biol. 2010; 6 (3): 238-243.

15. https://clinicaltrials.gov/ct2/home.

16. Munster P, Marchion D, Bicaku E, Lacevic M, Kim J, Centeno B et al. Clinical and biological effects of valproic acid as a histone deacetylase inhibitor on tumor and surrogate tissues: phase I/II trial of valproic acid and epirubicin/FEC. Clin Cancer Res. 2009; 15 (7): 2488-2496.

17. Vansteenkiste J, Van Cutsem E, Dumez H, Chen C, Ricker JL, Randolph SS et al. Early phase II trial of oral vorinostat in relapsed or refractory breast, colorectal, or non-small cell lung cancer. Invest New Drugs. 2008; 26 (5): 483-488.

18. Terranova-Barberio M, Pawlowska N, Dhawan M, Moasser M, Chien AJ, Melisko ME et al. Exhausted T cell signature predicts immunotherapy response in ERpositive breast cancer. Nat Commun. 2020; 11 (1): 3584.

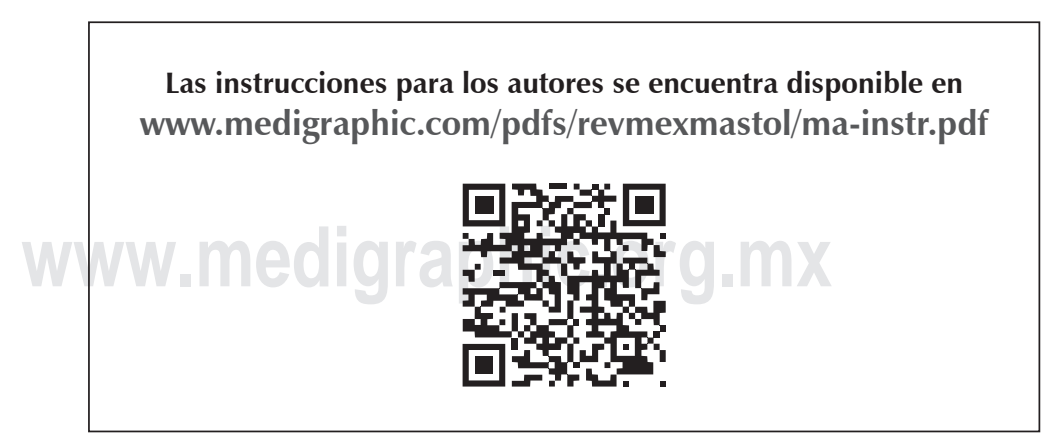

